\title{
Reduced expression of exosomal miR-29s in peritoneal fluid is a useful predictor of peritoneal recurrence after curative resection of gastric cancer with serosal involvement
}

\author{
HIDEYUKI OHZAWA ${ }^{1}$, AKIRA SAITO ${ }^{2}$, YUKO KUMAGAI ${ }^{2}$, YUKI KIMURA ${ }^{2}$, HIRONORI YAMAGUCHI ${ }^{2,3}$, \\ YOSHINORI HOSOYA ${ }^{2}$, ALAN KAWARAI LEFOR ${ }^{2}$, NAOHIRO SATA ${ }^{2}$ and JOJI KITAYAMA ${ }^{1,2}$ \\ ${ }^{1}$ Center for Clinical Research; Departments of ${ }^{2}$ Gastrointestinal Surgery and ${ }^{3}$ Clinical Oncology, \\ Jichi Medical University, Shimotsuke, Tochigi 329-0498, Japan
}

Received August 9, 2019; Accepted January 9, 2020

DOI: $10.3892 /$ or.2020.7505

\begin{abstract}
The peritoneal surface is the most frequent site of metastasis disease in patients with gastric cancer. Even after curative surgery and adjuvant chemotherapy, peritoneal recurrences often develop. Exosomes play pivotal roles in tumor metastasis via the transfer of microRNAs (miRNAs). In the present study, exosomes were isolated from peritoneal lavage fluid or ascites in 85 patients with gastric cancer and the relative expression levels of miR-29s were examined. The expression of miR-29a-3p, miR-29b-3p and miR-29c-3p in peritoneal exosomes were all downregulated in patients with peritoneal metastases (PM) compared to those without PM. In 30 patients who underwent curative gastrectomy with serosa-involved (T4) gastric cancer, 6 patients exhibited recurrence in the peritoneum within 12 months. The expression levels of miR-29s at gastrectomy tended to be lower in these 6 patients than in the other 24 patients with significant differences in miR-29b-3p ( $\mathrm{P}=0.003)$. When the patients were divided into two groups based on median levels of miR-29s, peritoneal recurrence developed more frequently in patients with low expression of miR-29b-3p, and lower expression of miR-29s were related with worse overall survival. miR-29s are thought to play a suppressive role in the growth of disseminated peritoneal tumor cells. Reduced expression of miR-29b in peritoneal exosomes is a strong risk factor of developing postoperative peritoneal recurrence.
\end{abstract}

\section{Introduction}

Gastric cancer is the fifth leading cause of cancer-related deaths worldwide (1), and peritoneal metastases (PM) are

Correspondence to: Professor Joji Kitayama, Department of Gastrointestinal Surgery, Jichi Medical University, Yakushiji 3311-1, Shimotsuke, Tochigi 329-0498, Japan

E-mail: kitayama@jichi.ac.jp

Key words: exosome, microRNA, miR-29, peritoneal metastasis, biomarker the most life-threatening form of recurrence $(2,3)$. Although various approaches have been used to treat PM such as extended surgery, chemotherapy, and heated intraperitoneal chemotherapy, the prognosis of patients with PM is still very poor (4-6). Even after curative surgery, approximately half of patients with gastric cancer with serosal involvement (T4) have been reported to develop peritoneal recurrences (7-9). Therefore, intensive adjuvant treatment to suppress peritoneal recurrence in high risk patients is a key factor to improve the survival of patients with advanced gastric cancer. However, no reliable biomarkers are available to accurately predict postoperative peritoneal recurrence in these patients, although molecular detection of CEA mRNA in peritoneal lavage fluid may have prognostic value (10-12).

Exosomes are small membrane-covered vesicles released from many different cell types and are present in various biological fluids. Recent studies have revealed that exosomes play pivotal roles in cell-to-cell communication via the transfer of protein, lipids, and nucleic acids, such as DNA, messenger RNA, microRNA (miRNA), and other non-coding RNAs (13). Accumulating evidence suggests that dysregulated expression of miRNAs with oncogenic or tumor-suppressing activities is often detected in patients with malignancies $(14,15)$. Recently, it has become evident that secretory exosomes contain numerous miRNAs and serve as an efficient vehicle for intercellular transfer of miRNA (16). Aberrant expression of exosomal miRNAs in serum can be used as potential biomarkers of tumor progression and metastasis $(17,18)$.

The miR-29 family is one of the most intensively examined groups of miRNAs. Previous studies have revealed that miR-29 plays an important role in various biological processes such as cell proliferation, apoptosis, invasion and angiogenesis and that downregulation of the miR-29 family is associated with aging, pathogenesis of neurodegenerative or fibrotic diseases, as well as tumor development and progression $(19,20)$. However, there is little information regarding miRNAs in peritoneal fluid. In this study, exosomes were isolated from peritoneal lavage fluid from patients with gastric cancer and it was determined that the relative expression levels of miR-29s in peritoneal exosomes were strongly associated with the development of PM. 


\section{Materials and methods}

Patients and sample collection. The study protocol was approved by the Bioethics Committee for Clinical Research A, Jichi Medical University Hospital (Approval \#A15-163), and written informed consent was obtained from all participants. All patients had pathologically confirmed gastric cancer in biopsy specimens obtained endoscopically from a primary lesion. Peritoneal fluid samples were collected from 85 patients (median age 69, range, 35-86; 49 male and 36 female patients) who were treated at Jichi Medical University Hospital between January 2016 and June 2019. Peritoneal lavage fluid was obtained from patients who underwent open surgery just after laparotomy. All of the patients from whom peritoneal lavage fluids were collected had fairly good performance status and received elective surgery. Patients who received urgent surgery, such as gastrointestinal perforation and acute peritonitis, were excluded from this study. In some patients with PM, malignant ascites were obtained by paracentesis or laparoscopic examination under general anesthesia. The present study was performed in accordance with the precepts established by the Helsinki Declaration.

Isolation of extracellular vesicles and RNA extraction. Peritoneal fluid samples were centrifuged at 2,000 x g for $10 \mathrm{~min}$ to remove floating cells. Supernatants were filtered through an 800-nm filter (EMD Millipore) to remove cell debris. For exosome preparation, filtered samples were ultracentrifuged at $150,000 \mathrm{x}$ g for $70 \mathrm{~min}$ at $4^{\circ} \mathrm{C}$. The size distribution and number of exosomes were determined using Nanosight LM10 (Malvern Panalytical, Ltd.). RNA extraction was performed using an miRNeasy Mini kit (Qiagen $\mathrm{GmbH}$ ) according to the manufacturer's instructions. Total RNA in the samples was verified for quantity and quality using an Agilent 2100 Bioanalyzer (Agilent Technologies, Inc.).

Western blot analysis. Protein concentration of exosome fraction was determined using a Qubit Protein Assay Kit with Qubit 2.0 Fluorometer (Invitrogen; Thermo Fisher Scientific, Inc.). The exosome fractions (1 $\mu \mathrm{g}$ of protein) were directly mixed with loading buffer and heated at $70^{\circ} \mathrm{C}$ for $10 \mathrm{~min}$ as previously described (21) and loaded and separated using 10\% Novex Bis-Tris Protein Gels (Invitrogen; Thermo Fisher Scientific, Inc.), and then transferred onto PVDF membranes (Invitrogen), and immunoblotted with the following primary antibodies (incubated overnight at $4^{\circ} \mathrm{C}$ ): CD9 (dilution 1:200; cat. no. sc-59140; Santa Cruz Biotechnology, Inc.) and CD63 (dilution 1:1,000; cat. no. MEX002-3; Medical \& Biological Laboratories). Anti-mouse IgG, peroxidase-linked antibody (dilution 1:50,000; cat. no. NA931; GE Healthcare) was used as a secondary antibody (incubated for $1 \mathrm{~h}$ at room temprature). Chemiluminescence was detected using Amersham ECL Prime Western Blotting Detection Reagents (GE Healthcare Bio-Sciences) and imaged using LAS-3000 mini (Fujifilm Life Sciences).

miRNA expression analysis. In the initial screening phase, pooled samples from patients with or without PM were analyzed using miScript SYBR Green PCR Kit and the Human miRNome miScript miRNA PCR Array (Qiagen
$\mathrm{GmbH}$ ). A custom miScript miRNA PCR Array (Qiagen $\mathrm{GmbH}$ ) was constructed based on the results. The miRNA expression profiles in each sample were evaluated using the custom PCR array. Using the NormFinder algorithm (22), miR-30d-5p and miR-10a-5p were identified as the most stably expressed miRNAs, which were selected as internal controls. Then, 8 miRNAs and 3 miRNAs (miR29a-3p, miR-29b-3p, and $\operatorname{miR} 29 \mathrm{c}-3 \mathrm{p}$ ) were significantly up- and downregulated in samples with PM, respectively. The miR-29 family was selected for further validation since all three miRNAs were downregulated in samples with PM.

For the quantitative PCR reaction, complementary DNA (cDNA) templates were prepared from $2 \mathrm{ml}$ of total RNA using TaqMan Advanced miRNA cDNA Synthesis Kit (Thermo Fisher Scientific, Inc.) according to the manufacturer's instructions. A quantitative PCR reaction was subsequently performed in triplicate with a 1:10 dilution of cDNA using TaqMan Advanced miRNA Assays (Thermo Fisher Scientific, Inc.) on a ViiA7 Real-Time PCR system (Applied Biosystems; Thermo Fisher Scientific, Inc.). Relative expression levels of miR-29a-3p, miR-29b-3p, and miR-29c-3p were determined using relative quantification analysis module on Thermo Fisher Cloud (Thermo Fisher Scientific, Inc.). All miRNA data were calculated relative to the average of miR-10a-5p and miR-30d-5p performed on the same reaction plate.

Statistical analysis. The difference between categorical variables was evaluated using chi-square and Fisher's exact test. Relative expression values obtained from each PCR experiment were compared with linear regression analysis and the Mann-Whitney or Kruskal-Wallis test. Survival curves were calculated using the Kaplan-Meier method and differences were evaluated using the log-rank test. Patients were divided into miR-29s 'high' and 'low' groups based on the median expression values. Statistical analysis was conducted using GraphPad Prism 8.2.0 (GraphPad Software, Inc.), and differences were considered statistically significant with $\mathrm{P}<0.05$.

\section{Results}

Characteristics of exosomes isolated from peritoneal fluid. Peritoneal fluid was collected from 33 patients with PM and 52 patients without $\mathrm{PM}$, and exosomes were isolated by ultracentrifugation. Nanotracking analysis revealed that the isolated exosomes were mostly between 100 and $200 \mathrm{~nm}$ in size and western blotting revealed the presence of CD9 and CD63 (Fig. 1). Although the expression levels of the exosome markers varied among samples possibly due to the different protein composition in peritoneal fluids, these results clarified that we were working with exosomes. The protein concentration of the exosome fractions was higher in samples from patients with PM compared to those without PM (protein concentration $(\mathrm{mg} / \mathrm{ml})$, median $=4.66$, range: $0.16-20.6$ vs. median $=0.71,0.17-1.52$ ). However, the number as well as the size distribution of the exosomes were almost the same in both groups [particle size $(\mathrm{nm})$, median $=136.5$, range: 93.0-175.0 vs. median=157.9, range: $120.7-252.9$, number of particles $\left(x 10^{10}\right.$ particles $\left./ \mathrm{ml}\right)$, median $=30.3$, range: $14.3-112.2$ vs. median $=23.5$, range: $2.2-104.0]$. 

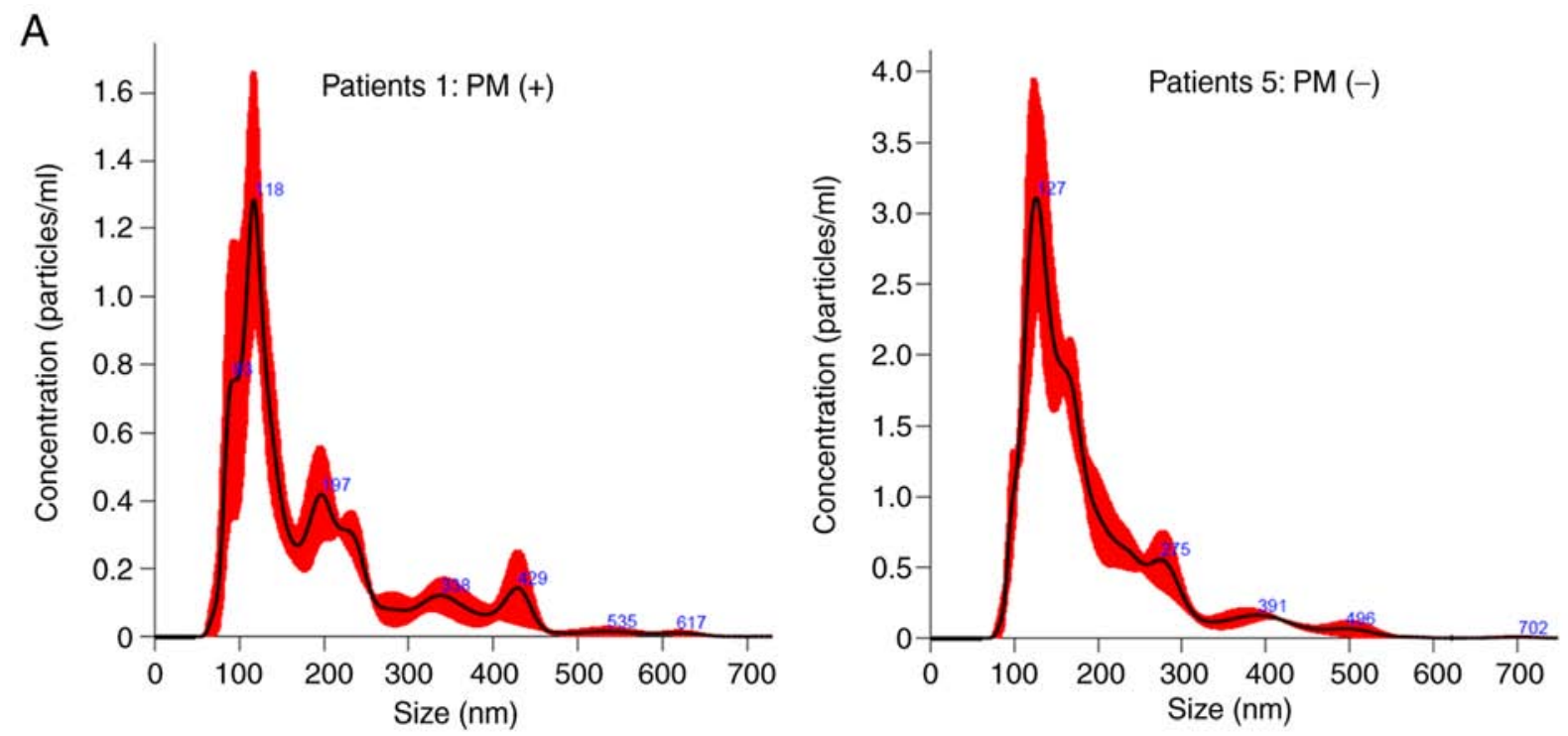

B $\quad \mathrm{MW}(\mathrm{kDa})$
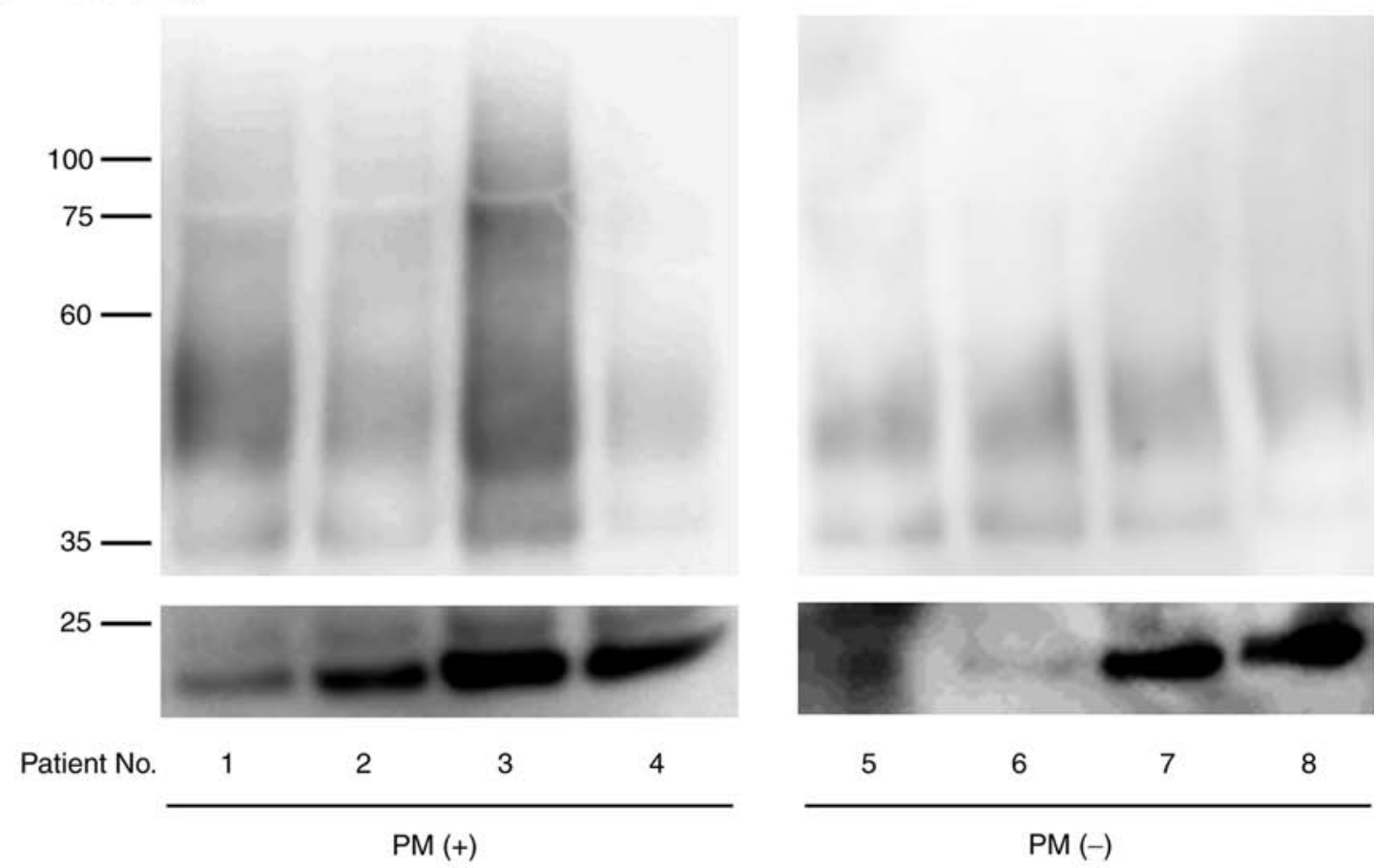

Figure 1. Representative features of exosomes purified from peritoneal fluid obtained from patients with and without peritoneal metastases. (A) Nanoparticle tracking analysis (B) Western blotting for CD9 or CD63. PM, peritoneal metastases.

The miR-29 family is downregulated in exosome in peritoneal fluids with $P M$. Relative expression levels of miR-29a-3p, miR-29b-3p and miR-29c-3p extracted from the exosome fractions were evaluated against an internal control, which revealed a positive correlation among the 3 miRs (Fig. 2). As revealed in Fig. 3, in patients without PM, the relative expression levels of these miR-29s were not different among tumors with serosal exposure (T4) and less invasive tumors (T1-T3) $(\mathrm{miR}-29 \mathrm{a}-3 \mathrm{p}$, median $=2.55$, range: $1.69-8.29$ vs. median $=2.53$, range: $1.35-4.42$; miR-29b-3p, median $=4.34$, range: $2.49-12.76$ vs. median $=3.73$, range: $1.48-8.55$; miR-29c-3p, median $=3.02$, range: $1.31-9.58$ vs. median=2.99, range: $0.92-6.67)$. However, their expression levels were significantly reduced in samples from patients with PM compared with those from patients without PM (miR-29a-3p, median=1.75, range: 0.53-5.07, $\mathrm{P}<0.001$; miR-29b-3p, median=2.02, range: $0.32-4.56, \mathrm{P}<0.001$; miR-29c-3p, median $=1.37$, range: $0.24-2.84, \mathrm{P}<0.001)$.

Low miR-29b-3p expression in peritoneal exosomes is associated with peritoneal recurrence in patients with T4 gastric cancer. Next, the expression of the miR-29 family and outcomes of the 30 patients with T4 tumors with serosal involvement were examined. Among them, 24 patients did not develop peritoneal recurrence with a median follow-up of 24 months, whereas peritoneal recurrence was observed with radiologic 

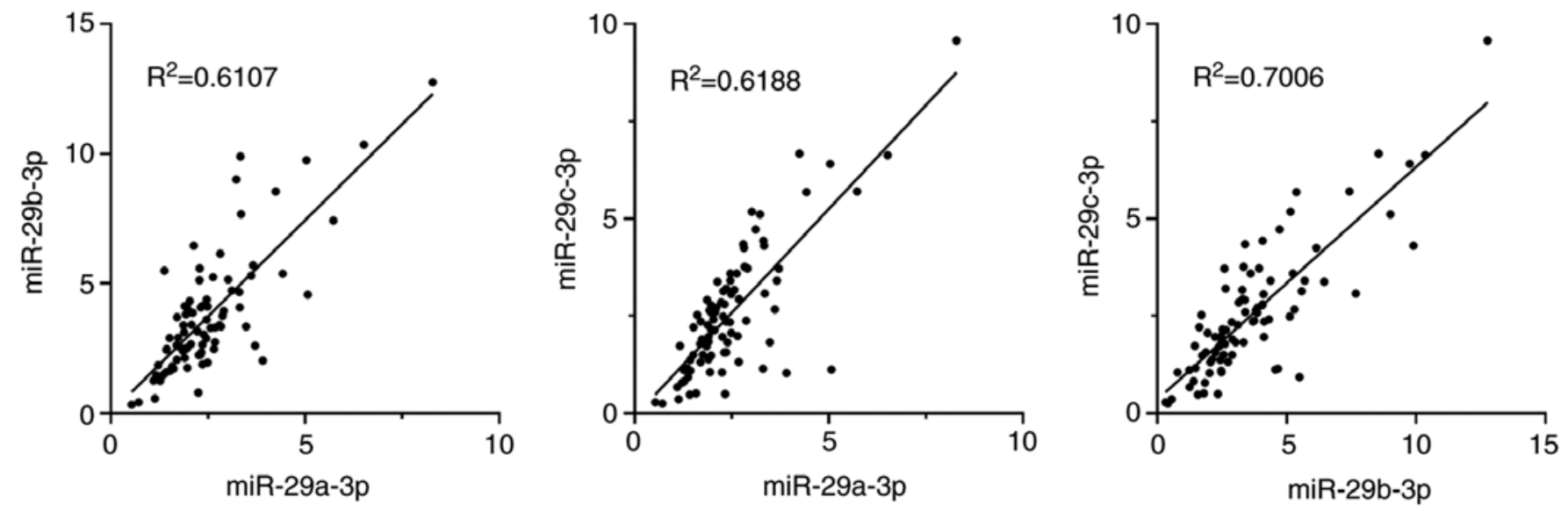

Figure 2. Correlation of the expression levels of miR-29a-3p, miR-29b-3p and miR-29c-3p in exosomes derived from the peritoneal fluid of all 85 patients. $\mathrm{R}^{2}$ and P-values were examined using Spearman's correlation analysis.
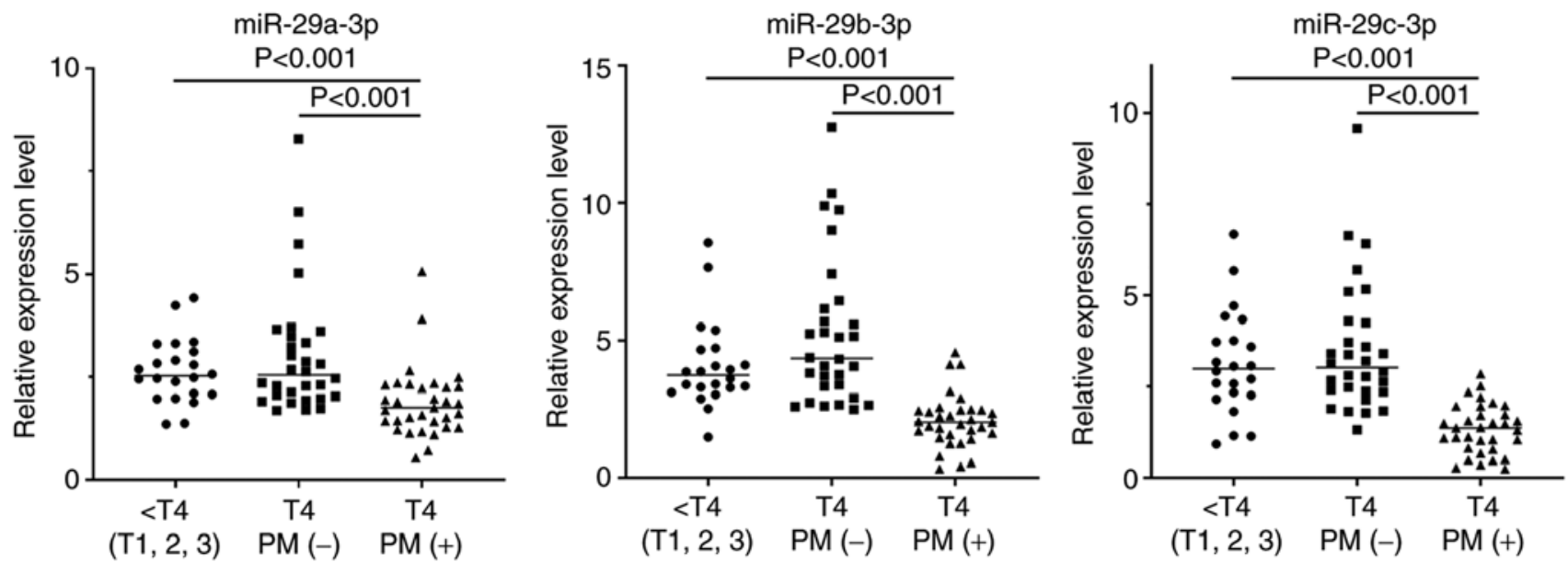

Figure 3. Expression levels of miR-29a-3p, miR-29b-3p and miR-29c-3p in 33 patients with PM as well as 52 patients without PM which were divided into T1-3 $(n=22)$ and T4 $(n=30)$ tumors. P-values were analyzed by the Kruskal-Wallis test. Relative expression levels were calculated relative to an average of miR-10a-5p and miR-30d-5p. PM, peritoneal metastases.

findings in the other 6 patients within 12 months after curative gastrectomy. Clinical and pathological factors revealed no significant differences comparing patients with and without recurrent disease (Table I). However, the expression levels of miR-29b-3p and miR-29c-3p in peritoneal exosomes obtained at surgery were significantly lower in the 6 patients with peritoneal recurrence compared with the other 24 patients (miR-29b-3p, median $=2.82$, range: $2.59-3.75$ vs. median $=5.19$, range: $2.49-12.76, \mathrm{P}=0.003$; miR-29c-3p, median=2.13, range: 1.31-3.71 vs. median $=3.29$, range: $1.81-9.58, \mathrm{P}=0.038$ ) (Fig. 4). The level of miR-29a-3p also exhibited a similar trend although the differences were not statistically significant. When the patients were divided into two groups based on median levels of miR-29s, the peritoneal recurrence-free survival of patients with low expression of miR-29b-3p were significantly worse than those of patients with high expression of miR-29b-3p $(\mathrm{P}=0.014)$. The overall survival of patients with low expression of miR-29a-3p, miR-29b-3p, and miR-29c-3p was significantly worse than those of patients with high expression of these miRNAs, respectively. (miR-29a-3p, $\mathrm{P}=0.038$; miR-29b-3p, $\mathrm{P}=0.031$; miR-29c-30, $\mathrm{P}=0.021$ ) (Fig. 5).

\section{Discussion}

miRNAs are non-coding RNAs with $<25$ nucleotides which have important roles in a wide range of pathophysiological processes through post-transcriptional gene repression of target RNA transcripts. In particular, miRNAs in exosomes circulating in blood or body fluids are resistant to enzymatic degradation by RNase and play pivotal roles in tumor metastasis formation $(14,15)$. Emerging evidence has demonstrated that dysregulation of miRNAs has been revealed to be related to tumor development and progression $(17,18)$, and that exosomes and exosomal miRNAs in circulating blood can be used as non-invasive biomarkers for early diagnosis and evidence of tumor progression in various malignancies (23-26), including gastric cancer $(27,28)$.

However, little information is available regarding the miRNA profile in peritoneal exosomes, although 5 miRNAs have been revealed to be upregulated in malignant ascites, which could be candidate miRNA species related to peritoneal dissemination in gastric cancer (29). In patients with PM, however, tumor nodules were directly exposed to peritoneal 
Table I. Characteristics of patients with pT4 gastric cancer with or without peritoneal recurrence.

\begin{tabular}{|c|c|c|c|}
\hline Variables & $\begin{array}{c}\text { Peritoneal } \\
\text { recurrence }(+)\end{array}$ & $\begin{array}{l}\text { Peritoneal } \\
\text { recurrence (-) }\end{array}$ & P-values \\
\hline Age & & & N.S. \\
\hline Median (min-max) & 70 (57 to 82$)$ & 69 (35 to 86$)$ & \\
\hline Sex & & & N.S. \\
\hline Male & 3 & 14 & \\
\hline Female & 3 & 10 & \\
\hline Location & & & N.S. \\
\hline Upper & 3 & 9 & \\
\hline Middle & 1 & 7 & \\
\hline Lower & 2 & 8 & \\
\hline Macroscopic type & & & N.S. \\
\hline 2 & 3 & 4 & \\
\hline 3 & 2 & 16 & \\
\hline 4 & 1 & 3 & \\
\hline Other & 0 & 1 & \\
\hline Histologic type & & & N.S. \\
\hline Differentiated & 2 & 16 & \\
\hline Undifferentiated & 4 & 8 & \\
\hline Venous invasion & & & N.S. \\
\hline+ & 6 & 24 & \\
\hline- & 0 & 0 & \\
\hline Lymphatic invasion & & & N.S. \\
\hline+ & 5 & 24 & \\
\hline- & 1 & 0 & \\
\hline $\mathrm{pT}$ & & & N.S. \\
\hline pT4a & 6 & 23 & \\
\hline pT4b & 0 & 1 & \\
\hline pN stage & & & N.S. \\
\hline pNO & 0 & 1 & \\
\hline $\mathrm{pN} 1$ & 0 & 4 & \\
\hline $\mathrm{pN} 2$ & 3 & 7 & \\
\hline pN3 & 3 & 12 & \\
\hline pStage & & & N.S. \\
\hline IIB & 0 & 1 & \\
\hline IIIA & 0 & 5 & \\
\hline IIIB & 3 & 7 & \\
\hline IIIC & 3 & 11 & \\
\hline CEA (ng/ml) & & & N.S. \\
\hline Median (min-max) & $5.0(0.5-357.3)$ & $2.3(1.0-29.5)$ & \\
\hline CA19-9 (U/ml) & & & N.S. \\
\hline Median (min-max) & $\begin{array}{c}13.0 \\
(2.0-616.0)\end{array}$ & $\begin{array}{c}13.5 \\
(2.0-155.0)\end{array}$ & \\
\hline Operative procedure & & & N.S. \\
\hline Total gastrectomy & 4 & 15 & \\
\hline Partial gastrectomy & 2 & 9 & \\
\hline Adjuvant therapy & & & N.S. \\
\hline+ & 5 & 16 & N.S. \\
\hline- & 1 & 8 & \\
\hline
\end{tabular}

N.S., not significant; CEA, carcinoembryonic antigen. surface and numerous tumor cells were contained in peritoneal fluids, at least, more than circulating blood. Therefore, it is supposed that large amounts of exosomes containing tumor specific molecules including functional miRNAs are released in the peritoneal cavity before the formation of PM, and thus analysis of the miRNA profile in peritoneal exosomes should provide important information to develop the adequate treatment of PM.

The number as well as size distribution of the exosomes were almost the same between $\mathrm{PM}(+)$ and (-) patients, although the protein concentration of the exosome fractions was higher in samples from $\mathrm{PM}(+)$ patients, possibly due to the contaminated protein complexes in peritoneal fluids. However, the present results clearly revealed that exosomal miR-29s in peritoneal fluid were significantly downregulated in patients with PM. Moreover, patients with T4 tumors with serosal involvement who had low levels of miR-29b-3p in peritoneal fluid obtained at gastrectomy recurred frequently in the peritoneal space. In the present study, although the sample size was not large, this was the only significant predictor of peritoneal recurrence since other clinical and pathological factors did not have a positive correlation.

The expression levels of miR-29b-3p and miR-29c-3p were even lower in patients with PM compared with recurred patients with non-PM. Patients with PM had macroscopic metastasis, and recurred patients with non-PM may have residual tumor cells which cannot be detected either by macroscopic observation or conventional cytology. Therefore, the expression levels of miR-29s in exosomes may reflect the tumor burden in peritoneum.

miR-29s predominantly function as tumor suppressors $(30,31)$. In gastric cancer, miR-29 has been revealed to downregulate CCND2 and MMP-2 (32) or DNMT3A (33) which are critically involved in carcinogenesis and tumor progression, and their expression levels have been revealed to be reduced in gastric cancer tissues as compared with adjacent non-malignant tissue $(32,34,35)$. Moreover, Zhang et al revealed that miR-29a/c derived from cell-derived microvesicles suppressed angiogenesis and inhibited the growth of gastric cancer (36). Serum levels of miR-29s have been revealed to be reduced in glioma (37) and hepatocellular carcinoma (38). The data in the present study were consistent with those results, and suggest that miR-29s in peritoneal exosomes have biological relevance for the development of PM.

Recently, Wei et al also revealed that exosomes derived from human malignant ascites converted mesothelial cells to carcinoma-associated fibroblasts and induced peritoneal fibrosis and promoted PM (39). Deng et al revealed that gastric cancer cells produced exosomes which promoted the formation of PM by disrupting the mesothelial barrier and inducing fibrosis (40). These facts suggest that exosomes in malignant ascites may promote peritoneal fibrosis which appears to be a favorable microenvironment for disseminated tumor cells. In contrast, it is well known that miR-29s target multiple genes encoding extracellular matrix components such as collagen and fibronectin, and that loss of miR-29 contributes to fibrosis in various organs (41-43). In fact, Yu et al demonstrated in a murine model that in vivo gene transfer of miR-29b using an ultrasound-microbubble technique effectively inhibited dialysis-related peritoneal fibrosis through the blockade of the Sp1-TGF-b/Smad pathway (44). These facts 


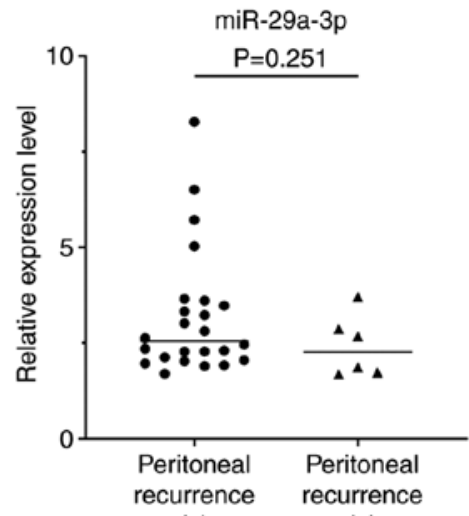

$(-)$

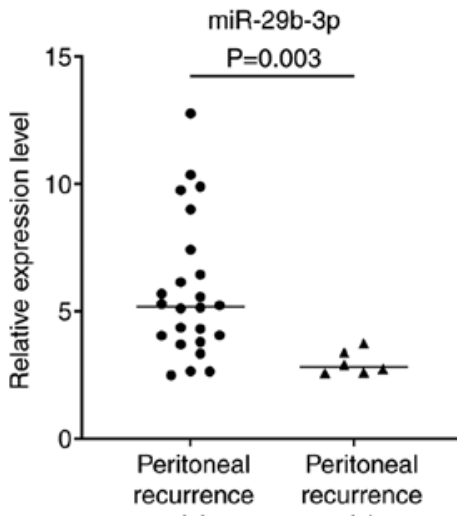

$(-)$

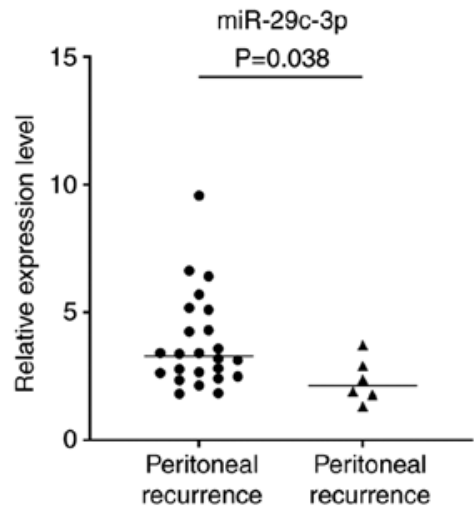

$(+)$

Figure 4. Expression levels of miR-29a-3p, miR-29b-3p and miR-29c-3p in 30 patients with T4 tumors who both had peritoneal recurrences (n=6) after curative resection and who did not $(n=24)$. P-values were evaluated with the Mann-Whitney test. Relative expression levels were calculated relative to an average of miR-10a-5p and miR-30d-5p.

\section{A}

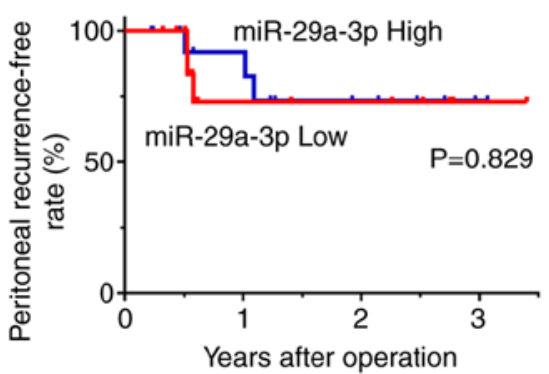

D

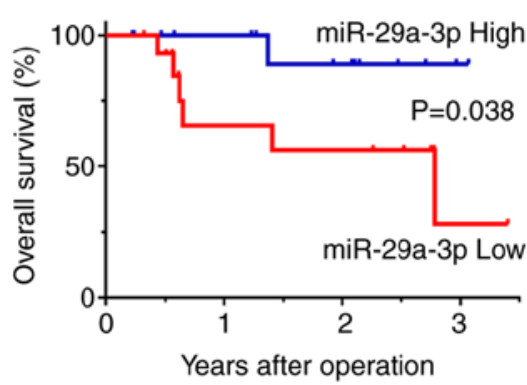

B

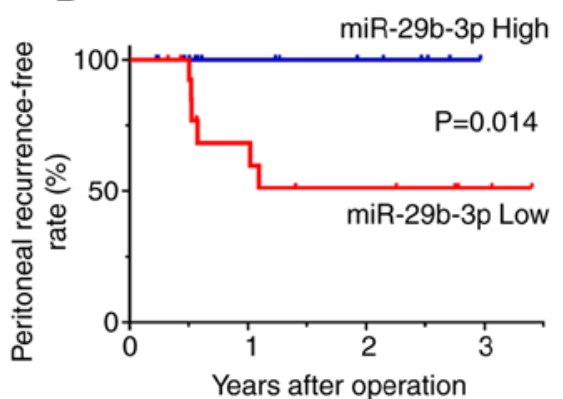

E

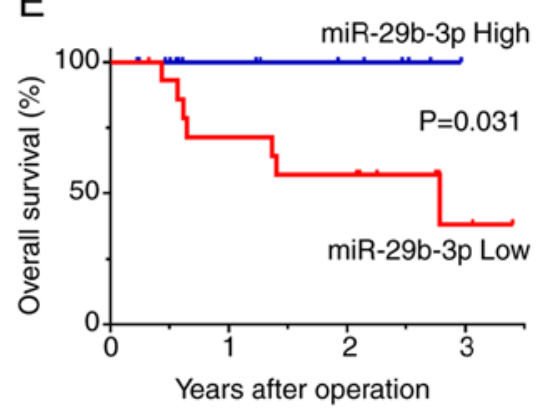

C

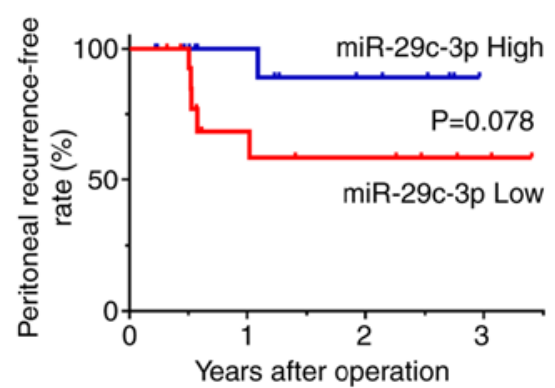

F

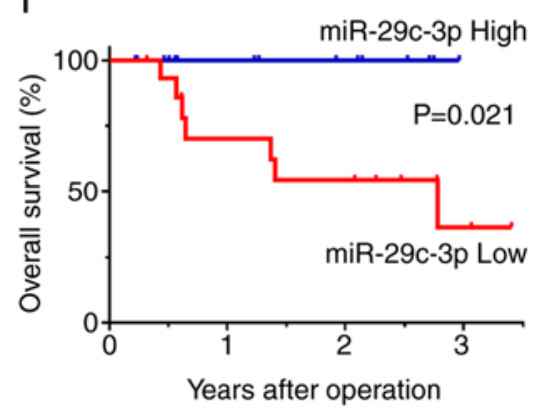

Figure 5. Clinical outcomes of patients with T4 tumors who underwent curative gastrectomy of the T4 tumor. Patients were divided into miR-29s 'high' and 'low' groups based on the median values. Survival rates were calculated using the Kaplan-Meier method and differences were evaluated using the log-rank test. Peritoneal (A-C) recurrence-free survival and (D-F) overall survival in patients with low and high expression of miR-29a-3p, miR-29b-3p, miR-29c-3p respectively.

indicated that miR-29s in peritoneal exosomes have the opposite effect and act as a defender against the progression of peritoneal fibrosis which favors the pro-metastatic niche. Further studies will be undertaken to clarify the mechanism of miR-29s and peritoneal metastasis.

There are some limitations to this study that should be addressed. First, the sample size was relatively small and examined at a single institution. Second, CD9 and CD63, which were generally used as exosome markers, were confirmed with western blotting, but other subpopulation markers for extracellular vesicles, such as TyA and C1a for ectosomes, were not verified in this study. The presence of other larger vesicles could not be excluded.
In summary, it was revealed that the level of miR-29s in peritoneal lavage-derived exosomes was a reliable biomarker to predict peritoneal recurrence in patients who underwent curative surgery for T4 gastric cancer. Reduction of miR-29 levels in peritoneal fluid may be a crucial event for the development of PM. In Asia, postoperative administration of S-1 or capecitabine and oxaliplatin is standard adjuvant treatment for curatively resected stage II/III gastric cancer $(45,46)$. However, these adjuvant therapies do not adequately suppress peritoneal recurrence, especially in patients with T4 tumors, and more intense adjuvant treatment should be considered for selected patients in the high-risk group. Recent advances in nanotechnology suggest that exosomes could be used as an 
effective tool for gene delivery and targeted therapy $(47,48)$. Collectively, these data indicated that miR-29s are good therapeutic targets in patients with PM and that replacement of exosomal miR-29b-3p in the peritoneal cavity may be a novel strategy to prevent peritoneal recurrence in patients with low expression.

\section{Acknowledgements}

We thank Drs Shiro Matsumoto, Hidenori Haruta, Kentaro Kurashina, Shin Saito for the help of sample acquisition. We also thank Professor Takahiro Ochiya and Dr Yusuke Yoshioka for their qualified advice on exosome isolation and analysis.

\section{Funding}

This publication was subsidized by JKA through its promotion funds from KEIRIN RACE. The present study was supported by a Japan Society for the Promotion of Science (17H04286) and the Japan Agency of Medical Research and Development (17lk0201053h0002).

\section{Availability of data and materials}

The datasets used and/or analyzed during the current study are available from the corresponding author on reasonable request.

\section{Authors' contributions}

$\mathrm{HO}$ and $\mathrm{JK}$ designed the project. $\mathrm{HO}, \mathrm{AS}, \mathrm{YKu}$ and $\mathrm{YKi}$ performed the experiments. HY, YH and NS contributed to the analysis and interpretation of the data. HO drafted the work. AKL and JK wrote the paper and revised it critically. All authors read and approved the manuscript and agree to be accountable for all aspects of the research in ensuring that the accuracy or integrity of any part of the work are appropriately investigated and resolved.

\section{Ethics approval and consent to participate}

The study protocol was approved by the Bioethics Committee for Clinical Research A, Jichi Medical University Hospital (Approval \#A15-163), and written informed consent was obtained from all participants.

\section{Patient consent for publication}

Not applicable.

\section{Competing interests}

All authors declare that they have no competing interests.

\section{References}

1. GLOBOCAN 2012: Estimated Cancer Incidence, Mortality and Prevalence Worldwide in 2012. v1.0. IARC CancerBase No. 11. IARC, Lyon, 2012.

2. Jemal A, Bray F, Center MM, Ferlay J, Ward E and Forman D: Global cancer statistics. CA Cancer J Clin 61: 69-90, 2011.
3. Nashimoto A, Akazawa K, Isobe Y, Miyashiro I, Katai H, Kodera Y, Tsujitani S, Seto Y, Furukawa H, Oda I, et al: Gastric cancer treated in 2002 in Japan: 2009 annual report of the JGCA nationwide registry. Gastric Cancer 16: 1-27, 2013.

4. Tan HL, Chia CS, Tan GH, Choo SP, Tai DW, Chua CW, Ng MC, Soo KC and Teo MC: Gastric peritoneal carcinomatosis-a retrospective review. World J Gastrointest Oncol 9: 121-128, 2017.

5. Dahdaleh FS and Turaga KK: Evolving treatment strategies and outcomes in advanced gastric cancer with peritoneal metastasis. Surg Oncol Clin N Am 27: 519-537, 2018.

6. Kitayama J, Ishigami H, Yamaguchi H, Sakuma Y, Horie H, Hosoya Y, Lefor AK and Sata N: Treatment of patients with peritoneal metastases from gastric cancer. Ann Gastroenterol Surg 2: 116-123, 2018.

7. Fujimoto S, Takahashi M, Mutou T, Kobayashi K, Toyosawa T, Isawa $\mathrm{E}$, Sumida $\mathrm{M}$ and Ohkubo $\mathrm{H}$ : Improved mortality rate of gastric carcinoma patients with peritoneal carcinomatosis treated with intraperitoneal hyperthermic chemoperfusion combined with surgery. Cancer 79: 884-891, 1997.

8. Kuramoto M, Shimada S, Ikeshima S, Matsuo A, Yagi Y, Matsuda M, Yonemura Y and Baba H: Extensive intraoperative peritoneal lavage as a standard prophylactic strategy for peritoneal recurrence in patients with gastric carcinoma. Ann Surg 250: 242-246, 2009.

9. Zhu BY, Yuan SQ, Nie RC, Li SM, Yang LR, Duan JL, Chen YB and Zhang XS: Prognostic factors and recurrence patterns in T4 gastric cancer patients after curative resection. J Cancer 10: 1181-1188, 2019.

10. Kodera Y, Nakanishi H, Ito S, Yamamura Y, Kanemitsu Y, Shimizu Y, Hirai T, Yasui K, Kato $\mathrm{T}$ and Tatematsu M: Quantitative detection of disseminated free cancer cells in peritoneal washes with real-time reverse transcriptase-polymerase chain reaction: A sensitive predictor of outcome for patients with gastric carcinoma. Ann Surg 235: 499-506, 2002.

11. Wang JY, Lin SR, Lu CY, Chen CC, Wu DC, Chai CY, Chen FM, Hsieh JS and Huang TJ: Gastric cancer cell detection in peritoneal lavage: RT-PCR for carcinoembryonic antigen transcripts versus the combined cytology with peritoneal carcinoembryonic antigen levels. Cancer Lett 223: 129-135, 2005.

12. Fujiwara Y, Doki Y, Taniguchi H, Sohma I, Takiguchi S, Miyata $\mathrm{H}$, Yamasaki $\mathrm{M}$ and Monden M: Genetic detection of free cancer cells in the peritoneal cavity of the patient with gastric cancer: Present status and future perspectives. Gastric Cancer 10: 197-204, 2007.

13. van Niel G, D'Angelo G and Raposo G: Shedding light on the cell biology of extracellular vesicles. Nat Rev Mol Cell Biol 19: 213-228, 2018.

14. Lu J, Getz G, Miska EA, Alvarez-Saavedra E, Lamb J, Peck D, Sweet-Cordero A, Ebert BL, Mak RH, Ferrando AA, et al: MicroRNA expression profiles classify human cancers. Nature 435: 834-838, 2005.

15. Croce CM: Causes and consequences of microRNA dysregulation in cancer. Nat Rev Genet 10: 704-714, 2009.

16. Kosaka $\mathrm{N}$ and Ochiya $\mathrm{T}$ : Unraveling the mystery of cancer by secretory microRNA: Horizontal microRNA transfer between living cells. Front Genet 2: 97, 2011.

17. Matsumura T, Sugimachi K, Iinuma H, Takahashi Y, Kurashige J, Sawada G, Ueda M, Uchi R, Ueo H, Takano Y, et al: Exosomal microRNA in serum is a novel biomarker of recurrence in human colorectal cancer. Br J Cancer 113: 275-281, 2015.

18. Wang N, Wang L, Yang Y, Gong L, Xiao B and Liu X: A serum exosomal microRNA panel as a potential biomarker test for gastric cancer. Biochem Biophys Res Commun 493: 1322-1328, 2017.

19. Kwon JJ, Factora TD, Dey S and Kota J: A systematic review of miR-29 in cancer. Mol Ther Oncolytics 12: 173-194, 2019.

20. Alizadeh M, Safarzadeh A, Beyranvand F, Ahmadpour F, Hajiasgharzadeh K, Baghbanzadeh A and Baradaran B: The potential role of miR-29 in health and cancer diagnosis, prognosis, and therapy. J Cell Physiol 234: 19280-19297, 2019.

21. Witwer KW, Buzas EI, Bemis LT, Bora A, Lässer C, Lötvall J, Nolte-'t Hoen EN, Piper MG, Sivaraman S, Skog J, et al: Standardization of sample collection, isolation and analysis methods in extracellular vesicle research. J Extracell Vesicles 2: 20360, 2013.

22. Andersen CL, Jensen JL and Orntoft TF: Normalization of real-time quantitative reverse transcription-PCR data: A model-based variance estimation approach to identify genes suited for normalization, applied to bladder and colon cancer data sets. Cancer Res 64: 5245-5250, 2004. 
23. Kosaka N, Iguchi $\mathrm{H}$ and Ochiya T: Circulating microRNA in body fluid: A new potential biomarker for cancer diagnosis and prognosis. Cancer Sci 101: 2087-2092, 2010.

24. Joyce DP, Kerin MJ and Dwyer RM: Exosome-encapsulated microRNAs as circulating biomarkers for breast cancer. Int J Cancer 139: 1443-1448, 2016.

25. Toiyama Y, Okugawa Y, Fleshman J, Richard Boland C and Goel A: MicroRNAs as potential liquid biopsy biomarkers in colorectal cancer: A systematic review. Biochim Biophys Acta Rev Cancer 1870: 274-282, 2018.

26. Nedaeinia R, Manian M, Jazayeri MH, Ranjbar M, Salehi R, Sharifi M, Mohaghegh F, Goli M, Jahednia SH, Avan A, et al: Circulating exosomes and exosomal microRNAs as biomarkers in gastrointestinal cancer. Cancer Gene Ther 24: 48-56, 2017.

27. Shrestha S, Hsu SD, Huang WY, Huang HY, Chen W, Weng SL and Huang HD: A systematic review of microRNA expression profiling studies in human gastric cancer. Cancer Med 3: 878-888, 2014.

28. Link A and Kupcinskas J: MicroRNAs as non-invasive diagnostic biomarkers for gastric cancer: Current insights and future perspectives. World J Gastroenterol 24: 3313-3329, 2018.

29. Tokuhisa M, Ichikawa Y, Kosaka N, Ochiya T, Yashiro M, Hirakawa K, Kosaka T, Makino H, Akiyama H, Kunisaki C and Endo I: Exosomal miRNAs from peritoneum lavage fluid as potential prognostic biomarkers of peritoneal metastasis in gastric cancer. PLoS one 10: e0130472, 2015.

30. Mott JL, Kobayashi S, Bronk SF and Gores GJ: mir-29 regulates Mcl-1 protein expression and apoptosis. Oncogene 26: 6133-6140, 2007.

31. Fabbri M, Garzon R, Cimmino A, Liu Z, Zanesi N, Callegari E, Liu S, Alder H, Costinean S, Fernandez-Cymering C, et al MicroRNA-29 family reverts aberrant methylation in lung cancer by targeting DNA methyltransferases $3 \mathrm{~A}$ and 3B. Proc Natl Acad Sci USA 104: 15805-15810, 2007.

32. Gong J, Li J, Wang Y, Liu C, Jia H, Jiang C, Wang Y, Luo M, Zhao H, Dong L, et al: Characterization of microRNA-29 family expression and investigation of their mechanistic roles in gastric cancer. Carcinogenesis 35: 497-506, 2014.

33. Cui H, Wang L, Gong P, Zhao C, Zhang S, Zhang K, Zhou R, Zhao Z and Fan H: Deregulation between miR-29b/c and DNMT3A is associated with epigenetic silencing of the CDH1 gene, affecting cell migration and invasion in gastric cancer. PLoS One 10: e0123926, 2015.

34. Zhao X, Hou Y, Tuo Z and Wei F: Application values of miR-194 and miR-29 in the diagnosis and prognosis of gastric cancer. Exp Ther Med 15: 4179-4184, 2018.

35. Wang D, Fan Z, Liu F and Zuo J: Hsa-miR-21 and Hsa-miR-29 in tissue as potential diagnostic and prognostic biomarkers for gastric cancer. Cell Physiol Biochem 37: 1454-1462, 2015.

36. Zhang H, Bai M, Deng T, Liu R, Wang X, Qu Y, Duan J, Zhang L, Ning T, Ge S, et al: Cell-derived microvesicles mediate the delivery of miR-29a/c to suppress angiogenesis in gastric carcinoma. Cancer Lett 375: 331-339, 2016.

37. Wu J, Li L and Jiang C: Identification and Evaluation of Serum MicroRNA-29 Family for Glioma Screening. Mol Neurobiol 52: 1540-1546, 2015.
38. Parpart S, Roessler S, Dong F, Rao V, Takai A, Ji J, Qin LX, Ye QH, Jia HL, Tang ZY and Wang XW: Modulation of miR-29 expression by alpha-fetoprotein is linked to the hepatocellular carcinoma epigenome. Hepatology 60: 872-883, 2014

39. Wei M, Yang T, Chen X, Wu Y, Deng X, He W, Yang J and Wang Z: Malignant ascites-derived exosomes promote proliferation and induce carcinoma-associated fibroblasts transition in peritoneal mesothelial cells. Oncotarget 8: 42262-42271, 2017.

40. Deng G, Qu J, Zhang Y, Che X, Cheng Y, Fan Y, Zhang S, Na D, Liu Y and Qu X: Gastric cancer-derived exosomes promote peritoneal metastasis by destroying the mesothelial barrier. FEBS Lett 591: 2167-2179, 2017.

41. van Rooij E, Sutherland LB, Thatcher JE, DiMaio JM, Naseem RH, Marshall WS, Hill JA and Olson EN: Dysregulation of microRNAs after myocardial infarction reveals a role of miR-29 in cardiac fibrosis. Proc Natl Acad Sci USA 105: 13027-13032, 2008.

42. Roderburg C, Urban GW, Bettermann K, Vucur M, Zimmermann H, Schmidt S, Janssen J, Koppe C, Knolle P, Castoldi M, et al: Micro-RNA profiling reveals a role for miR-29 in human and murine liver fibrosis. Hepatology 53: 209-218, 2011.

43. Cushing L, Kuang PP, Qian J, Shao F, Wu J, Little F, Thannickal VJ, Cardoso WV and Lü J: miR-29 is a major regulator of genes associated with pulmonary fibrosis. Am J Respir Cell Mol Biol 45: 287-294, 2011.

44. Yu JW, Duan WJ, Huang XR, Meng XM, Yu XQ and Lan HY: MicroRNA-29b inhibits peritoneal fibrosis in a mouse model of peritoneal dialysis. Lab Invest 94: 978-990, 2014.

45. Sasako M, Sakuramoto S, Katai H, Kinoshita T, Furukawa H, Yamaguchi T, Nashimoto A, Fujii M, Nakajima T and Ohashi Y: Five-year outcomes of a randomized phase III trial comparing adjuvant chemotherapy with S-1 versus surgery alone in stage II or III gastric cancer. J Clin Oncol 29: 4387-4393, 2011.

46. Noh SH, Park SR, Yang HK, Chung HC, Chung IJ, Kim SW, Kim HH, Choi JH, Kim HK, Yu W, et al: Adjuvant capecitabine plus oxaliplatin for gastric cancer after D2 gastrectomy (CLASSIC): 5-year follow-up of an open-label, randomised phase 3 trial. Lancet Oncol 15: 1389-1396, 2014.

47. Wang X, Zhang H, Yang H, Bai M, Ning T, Li S, Li J, Deng T, Ying $\mathrm{G}$ and $\mathrm{Ba} \mathrm{Y}$ : Cell-derived exosomes as promising carriers for drug delivery and targeted therapy. Curr Cancer Drug Targets 18: 347-354, 2018.

48. Maheshwari R, Tekade M, Gondaliya P, Kalia K, D'Emanuele A and Tekade RK: Recent advances in exosome-based nanovehicles as RNA interference therapeutic carriers. Nanomedicine (Lond) 12: 2653-2675, 2017.

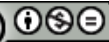

This work is licensed under a Creative Commons Attribution-NonCommercial-NoDerivatives 4.0 International (CC BY-NC-ND 4.0) License. 